\title{
On the fixed volume discrepancy of the Korobov point sets
}

\author{
A.S. Rubtsova*, K.S. Ryutin`, and V.N. Temlyakov ${ }^{\ddagger}$
}

\begin{abstract}
This paper is devoted to the study of a discrepancy-type characteristic - the fixed volume discrepancy - of the Korobov point sets in the unit cube. It was observed recently that this new characteristic allows us to obtain optimal rate of dispersion from numerical integration results. This observation motivates us to thoroughly study this new version of discrepancy, which seems to be interesting by itself. This paper extends recent results by $\mathrm{V}$. Temlyakov and M. Ullich on the fixed volume discrepancy of the Fibonacci point sets.
\end{abstract}

\section{Introduction}

This paper is a follow up to the recent paper [19]. It is devoted to the study of a discrepancy-type characteristic - the fixed volume discrepancy - of a point set in the unit cube $\Omega_{d}:=[0,1)^{d}$. We refer the reader to the following books and survey papers on discrepancy theory and numerical integration [2], [7, [9], [17], 3], [5], [14], and [18. Recently, an important new observation was made in [15. It claims that a new version of discrepancy - the $r$-smooth fixed volume discrepancy - allows us to obtain optimal rate of dispersion from numerical integration results (see [1, 4, 6, 10, 11, 12, 20, 21, 22, for some recent results on dispersion). This observation motivates us to thoroughly study this new version of discrepancy, which seems to be interesting by itself.

\footnotetext{
*Lomonosov Moscow State University, Russia.

${ }^{\dagger}$ Lomonosov Moscow State University, Russia.

$\ddagger$ University of South Carolina, USA; Steklov Institute of Mathematics and Lomonosov Moscow State University, Russia.
} 
The $r$-smooth fixed volume discrepancy takes into account two characteristics of a smooth hat function $h_{B}^{r}$-its smoothness $r$ and the volume of its support $v:=\operatorname{vol}(B)$ (see the definition of $h_{B}^{r}$ below). We now proceed to a formal description of the problem setting and to formulation of the results.

Denote by $\chi_{[a, b)}(x)$ a univariate characteristic function (on $\mathbb{R}$ ) of the interval $[a, b)$ and, for $r=1,2,3, \ldots$, we inductively define

$$
h_{u}^{1}(x):=\chi_{[-u / 2, u / 2)}(x)
$$

and

$$
h_{u}^{r}(x):=h_{u}^{r-1}(x) * h_{u}^{1}(x),
$$

where

$$
f(x) * g(x):=\int_{\mathbb{R}} f(x-y) g(y) d y .
$$

Note that $h_{u}^{2}$ is the hat function, i.e., $h_{u}^{2}(x)=\max \{u-|x|, 0\}$.

Let $\Delta_{t} f(x):=f(x)-f(x+t)$ be the first difference. We say that a univariate function $f$ has smoothness 1 in $L_{1}$ if $\left\|\Delta_{t} f\right\|_{1} \leq C|t|$ for some absolute constant $C<\infty$. In case $\left\|\Delta_{t}^{r} f\right\|_{1} \leq C|t|^{r}$, where $\Delta_{t}^{r}:=\left(\Delta_{t}\right)^{r}$ is the $r$ th difference operator, $r \in \mathbb{N}$, we say that $f$ has smoothness $r$ in $L_{1}$. Then, $h_{u}^{r}(x)$ has smoothness $r$ in $L_{1}$ and has support $(-r u / 2, r u / 2)$.

For a box $B$ of the form

$$
B=\prod_{j=1}^{d}\left[z_{j}-r u_{j} / 2, z_{j}+r u_{j} / 2\right)
$$

define

$$
h_{B}^{r}(\mathbf{x}):=h_{\mathbf{u}}^{r}(\mathbf{x}-\mathbf{z}):=\prod_{j=1}^{d} h_{u_{j}}^{r}\left(x_{j}-z_{j}\right) .
$$

We begin with the non-periodic $r$-smooth fixed volume discrepancy introduced and studied in [15].

Definition 1.1. Let $r \in \mathbb{N}, v \in(0,1]$ and $\xi:=\left\{\xi^{\mu}\right\}_{\mu=1}^{m} \subset[0,1)^{d}$ be a point set. We define the $r$-smooth fixed volume discrepancy with equal weights as

$$
D^{r}(\xi, v):=\sup _{B \subset \Omega_{d}: \operatorname{vol}(B)=v}\left|\int_{\Omega_{d}} h_{B}^{r}(\mathbf{x}) d \mathbf{x}-\frac{1}{m} \sum_{\mu=1}^{m} h_{B}^{r}\left(\xi^{\mu}\right)\right| .
$$


It is well known that the Fibonacci cubature formulas are optimal in the sense of order for numerical integration of different kind of smoothness classes of functions of two variables, see e.g. [5, 13, 17]. We present a result from [15], which shows that the Fibonacci point set has good fixed volume discrepancy.

Let $\left\{b_{n}\right\}_{n=0}^{\infty}, b_{0}=b_{1}=1, b_{n}=b_{n-1}+b_{n-2}, n \geq 2$, be the Fibonacci numbers. Denote the $n$th Fibonacci point set by

$$
\mathcal{F}_{n}:=\left\{\left(\mu / b_{n},\left\{\mu b_{n-1} / b_{n}\right\}\right): \mu=1, \ldots, b_{n}\right\} .
$$

In this definition $\{a\}$ is the fractional part of the number $a$. The cardinality of the set $\mathcal{F}_{n}$ is equal to $b_{n}$. In [15] we proved the following upper bound.

Theorem 1.1. Let $r \geq 2$. There exist constants $c, C>0$ such that for any $v \geq c / b_{n}$ we have

$$
D^{r}\left(\mathcal{F}_{n}, v\right) \leq C \frac{\log \left(b_{n} v\right)}{b_{n}^{r}}
$$

The main object of interest in the paper [19] was the periodic $r$-smooth $L_{p^{-}}$ discrepancy of the Fibonacci point sets. For this, we define the periodization $\tilde{f}$ (with period 1 in each variable) of a function $f \in L_{1}\left(\mathbb{R}^{d}\right)$ with a compact support by

$$
\tilde{f}(\mathbf{x}):=\sum_{\mathbf{m} \in \mathbb{Z}^{d}} f(\mathbf{m}+\mathbf{x})
$$

and, for each $B \subset[0,1)^{d}$, we let $\tilde{h}_{B}^{r}$ be the periodization of $h_{B}^{r}$ from (1.2).

We now define the periodic $r$-smooth $L_{p}$-discrepancy.

Definition 1.2. For $r \in \mathbb{N}, 1 \leq p \leq \infty$ and $v \in(0,1]$ define the periodic $r$-smooth fixed volume $L_{p}$-discrepancy of a point set $\xi$ by

$$
\tilde{D}_{p}^{r}(\xi, v):=\sup _{B \subset \Omega_{d}: \operatorname{vol}(B)=v}\left\|\int_{\Omega_{d}} \tilde{h}_{B}^{r}(\mathbf{x}-\mathbf{z}) d \mathbf{x}-\frac{1}{m} \sum_{\mu=1}^{m} \tilde{h}_{B}^{r}\left(\xi^{\mu}-\mathbf{z}\right)\right\|_{p}
$$

where the $L_{p}$-norm is taken with respect to $\mathbf{z}$ over the unit cube $\Omega_{d}=[0,1)^{d}$.

In the case of $p=\infty$ this concept was introduced and studied in [16]. The following upper bound for $1 \leq p<\infty$ was proved in [19]. 
Theorem 1.2. Let $r \in \mathbb{N}$ and $1 \leq p<\infty$. There exist constants $c, C>0$ such that for any $v \geq c / b_{n}$ we have

$$
\tilde{D}_{p}^{r}\left(\mathcal{F}_{n}, v\right) \leq C \frac{\sqrt{\log \left(b_{n} v\right)}}{b_{n}^{r}} .
$$

In the case $p=\infty$ a weaker upper bound was proved in [19].

Theorem 1.3. Let $r \geq 2$. There exist constants $c, C>0$ such that for any $v \geq c / b_{n}$ we have

$$
\tilde{D}_{\infty}^{r}\left(\mathcal{F}_{n}, v\right) \leq C \frac{\log \left(b_{n} v\right)}{b_{n}^{r}} .
$$

Some comments, which show that Theorems 1.2 and 1.3 cannot be improved in a certain sense were given in [19].

Our main interest in this paper is to study the Korobov cubature formulas instead of the Fibonacci cubature formulas from the point of view of the fixed volume discrepancy. We prove a conditional result under assumption that the Korobov cubature formulas are exact on a certain subspace of trigonometric polynomials with frequencies from a hyperbolic cross. There are results that guarantee existence of such cubature formulas (see Section 3 for a discussion).

Let $m \in \mathbb{N}, \mathbf{a}:=\left(a_{1}, \ldots, a_{d}\right), a_{1}, \ldots, a_{d} \in \mathbb{Z}$. We consider the cubature formulas

$$
P_{m}(f, \mathbf{a}):=m^{-1} \sum_{\mu=1}^{m} f\left(\left\{\frac{\mu a_{1}}{m}\right\}, \ldots,\left\{\frac{\mu a_{d}}{m}\right\}\right),
$$

which are called the Korobov cubature formulas. In the case $d=2, m=b_{n}$, $\mathbf{a}=\left(1, b_{n-1}\right)$ we have

$$
P_{m}(f, \mathbf{a})=\Phi_{n}(f):=\frac{1}{b_{n}} \sum_{\mathbf{y} \in \mathcal{F}_{n}} f(\mathbf{y}) .
$$

Denote

$$
\mathbf{y}^{\mu}:=\left(\left\{\frac{\mu a_{1}}{m}\right\}, \ldots,\left\{\frac{\mu a_{d}}{m}\right\}\right), \quad \mu=1, \ldots, m, \quad \mathcal{K}_{m}(\mathbf{a}):=\left\{\mathbf{y}^{\mu}\right\}_{\mu=1}^{m} .
$$

The set $\mathcal{K}_{m}(\mathbf{a})$ is called the Korobov point set. Further, denote

$$
S(\mathbf{k}, \mathbf{a}):=P_{m}\left(e^{i 2 \pi(\mathbf{k}, \mathbf{x})}, \mathbf{a}\right)=m^{-1} \sum_{\mu=1}^{m} e^{i 2 \pi\left(\mathbf{k}, \mathbf{y}^{\mu}\right)} .
$$


Note that

$$
P_{m}(f, \mathbf{a})=\sum_{\mathbf{k}} \hat{f}(\mathbf{k}) S(\mathbf{k}, \mathbf{a}), \quad \hat{f}(\mathbf{k}):=\int_{[0,1)^{d}} f(\mathbf{x}) e^{-i 2 \pi(\mathbf{k}, \mathbf{x})} d \mathbf{x},
$$

where for the sake of simplicity we may assume that $f$ is a trigonometric polynomial. It is clear that (1.6) holds for $f$ with absolutely convergent Fourier series.

It is easy to see that the following relation holds

$$
S(\mathbf{k}, \mathbf{a})=\left\{\begin{array}{lll}
1 & \text { for } & \mathbf{k} \in L(m, \mathbf{a}) \\
0 & \text { for } & \mathbf{k} \notin L(m, \mathbf{a})
\end{array}\right.
$$

where

$$
L(m, \mathbf{a}):=\{\mathbf{k}:(\mathbf{a}, \mathbf{k}) \equiv 0 \quad(\bmod m)\} .
$$

For $N \in \mathbb{N}$ define the hyperbolic cross by

$$
\Gamma(N, d):=\left\{\mathbf{k}=\left(k_{1}, \ldots, k_{d}\right) \in \mathbb{Z}^{d}: \prod_{j=1}^{d} \max \left(\left|k_{j}\right|, 1\right) \leq N\right\} .
$$

Denote

$$
\mathcal{T}(N, d):=\left\{f: f(\mathbf{x})=\sum_{\mathbf{k} \in \Gamma(N, d)} c_{\mathbf{k}} e^{i 2 \pi(\mathbf{k}, \mathbf{x})}\right\} .
$$

It is easy to see that the condition

$$
P_{m}(f, \mathbf{a})=\hat{f}(\mathbf{0}), \quad f \in \mathcal{T}(N, d),
$$

is equivalent to the condition

$$
\Gamma(N, d) \cap(L(m, \mathbf{a}) \backslash\{\mathbf{0}\})=\varnothing .
$$

Definition 1.3. We say that the Korobov cubature formula $P_{m}(\cdot, \mathbf{a})$ is exact on $\mathcal{T}(N, d)$ if condition (1.8) (equivalently, condition (1.9)) is satisfied.

Theorem 1.4. Suppose that $P_{m}(\cdot, \mathbf{a})$ is exact on $\mathcal{T}(L, d)$ with some $L \in \mathbb{N}$, $L \geq 2$. Let $r \in \mathbb{N}$ and $1 \leq p<\infty$. There exist constants $c(d), C(d, p)>0$ such that for any $v \geq c(d) / L$ we have

$$
\tilde{D}_{p}^{r}\left(\mathcal{K}_{m}(\mathbf{a}), v\right) \leq C(d, p) L^{-r}(\log (L v))^{(d-1) / 2} .
$$


In the case $p=\infty$ we prove a weaker upper bound.

Theorem 1.5. Let $r \geq 2$. Suppose that $P_{m}(\cdot, \mathbf{a})$ is exact on $\mathcal{T}(L, d)$ with some $L \in \mathbb{N}, L \geq 2$. There exist constants $c(d), C(d)>0$ such that for any $v \geq c(d) / L$ we have

$$
\tilde{D}_{\infty}^{r}\left(\mathcal{K}_{m}(\mathbf{a}), v\right) \leq C(d) L^{-r}(\log (L v))^{d-1} .
$$

\section{Proofs of Theorems 1.4 and 1.5}

The proofs of both theorems go along the same lines. We give a detailed proof of Theorem 1.4 and point out a change of this proof, which gives Theorem 1.5. For continuous functions of $d$ variables, which are 1-periodic in each variable, consider the Korobov cubature formula $P_{m}(\cdot, \mathbf{a})$.

For the univariate test functions $h_{u}^{r}$ we obtain by the properties of convolution that

$$
\hat{h}_{u}^{r}(y)=\hat{h}_{u}^{r-1}(y) \hat{h}_{u}^{1}(y), \quad y \in \mathbb{R}
$$

which implies for $y \neq 0$

$$
\hat{h}_{u}^{r}(y)=\left(\frac{\sin (\pi y u)}{\pi y}\right)^{r} .
$$

Therefore,

$$
\left|\hat{\tilde{h}}_{u}^{r}(k)\right| \leq \min \left(|u|^{r}, \frac{1}{|k|^{r}}\right)=\left(\frac{|u|}{k^{\prime}}\right)^{r / 2} \min \left(\left|k^{\prime} u\right|^{r / 2}, \frac{1}{|k u|^{r / 2}}\right),
$$

where $k^{\prime}:=\max \{1,|k|\}$. (Here, we used for a moment $\hat{h}$ for the Fourier transform of $h$ on $\mathbb{R}$. This should not lead to any confusion.)

It is convenient for us to use the following abbreviated notation for the product

$$
\operatorname{pr}(\mathbf{u}):=\operatorname{pr}(\mathbf{u}, d):=\prod_{j=1}^{d} u_{j} .
$$

For $B \subset \Omega_{d}$ of the form (1.1) and $\mathbf{z} \in \Omega_{d}$, we have

$$
\hat{\tilde{h}}_{B+\mathbf{z}}^{r}(\mathbf{k})=e^{-i 2 \pi(\mathbf{k}, \mathbf{z})} \hat{\tilde{h}}_{B}^{r}(\mathbf{k}),
$$


where $\tilde{h}_{B+\mathbf{z}}^{r}(\mathbf{x}):=\tilde{h}_{B}^{r}(\mathbf{x}-\mathbf{z})$, see (1.2). Therefore, we obtain from the above that

$$
\left|\hat{\tilde{h}}_{B}^{r}(\mathbf{k})\right| \leq \prod_{j=1}^{d}\left(\frac{\left|u_{j}\right|}{k_{j}^{\prime}}\right)^{r / 2} \min \left(\left|k_{j}^{\prime} u_{j}\right|^{r / 2}, \frac{1}{\left|k_{j} u_{j}\right|^{r / 2}}\right) .
$$

For $\mathbf{s} \in \mathbb{N}_{0}^{d}$, we define

$$
\rho(\mathbf{s}):=\left\{\mathbf{k} \in \mathbb{Z}^{d}:\left[2^{s_{j}-1}\right] \leq\left|k_{j}\right|<2^{s_{j}}, \quad j=1, \ldots, d\right\},
$$

where $[a]$ denotes the integer part of $a$, and obtain, for $\mathbf{k} \in \rho(\mathbf{s})$, that

$$
\left|\hat{\tilde{h}}_{B}^{r}(\mathbf{k})\right| \leq H_{B}^{r}(\mathbf{s}):=\left(\frac{p r(\mathbf{u})}{2^{\|\mathbf{s}\|_{1}}}\right)^{r / 2} \prod_{j=1}^{d} \min \left(\left(2^{s_{j}} u_{j}\right)^{r / 2}, \frac{1}{\left(2^{s_{j}} u_{j}\right)^{r / 2}}\right) .
$$

Later we will need certain sums of these quantities. First, consider

$$
\sigma_{\mathbf{u}}^{r}(t):=\sum_{\|\mathbf{s}\|_{1}=t} \prod_{j=1}^{d} \min \left(\left(2^{s_{j}} u_{j}\right)^{r / 2}, \frac{1}{\left(2^{s_{j}} u_{j}\right)^{r / 2}}\right), \quad t \in \mathbb{N}_{0} .
$$

The following technical lemma is part (I) from [15, Lemma 6.1].

Lemma 2.1. Let $r>0, t \in \mathbb{N}$ and $\mathbf{u} \in(0,1 / 2]^{d}$ be such that $\operatorname{pr}(\mathbf{u}) \geq 2^{-t}$. Then, we have

$$
\sigma_{\mathbf{u}}^{r}(t) \leq C(d) \frac{\left(\log \left(2^{t+1} \operatorname{pr}(\mathbf{u})\right)\right)^{d-1}}{\left(2^{t} \operatorname{pr}(\mathbf{u})\right)^{r / 2}}
$$

This lemma and (2.2) imply that

$$
\sum_{\|\mathbf{s}\|_{1}=t} H_{B}^{r}(\mathbf{s})^{2} \leq C_{1} 2^{-2 r t}\left(\log \left(2^{t} v\right)\right)^{d-1}
$$

where $v:=\operatorname{vol}(B)=r^{d} \operatorname{pr}(\mathbf{u})$, for all $r \geq 1$ and all $t \in \mathbb{N}_{0}$ with $v \geq r^{d} 2^{-t+1}$ and an absolute constant $C_{1}<\infty$.

Additionally, we need a result from harmonic analysis - a corollary of the Littlewood-Paley theorem. Denote

$$
\delta_{\mathbf{s}}(f, \mathbf{x}):=\sum_{\mathbf{k} \in \rho(\mathbf{s})} \hat{f}(\mathbf{k}) e^{i 2 \pi(\mathbf{k}, \mathbf{x})} .
$$


Then it is known that for $p \in[2, \infty)$ one has

$$
\|f\|_{p} \leq C(d, p)\left(\sum_{\mathbf{s} \in \mathbb{N}_{0}^{d}}\left\|\delta_{\mathbf{s}}(f)\right\|_{p}^{2}\right)^{1 / 2}
$$

Note that in the proof of Theorem 1.5 we use the simple triangle inequality

$$
\|f\|_{\infty} \leq \sum_{\mathbf{s}}\left\|\delta_{\mathbf{s}}(f)\right\|_{\infty}
$$

instead of (2.4).

Let us define

$$
E_{B}^{r}(\mathbf{z}):=\frac{1}{m} \sum_{\mu=1}^{m} \tilde{h}_{B}^{r}\left(\mathbf{y}^{\mu}-\mathbf{z}\right)-\int_{[0,1)^{d}} \tilde{h}_{B}^{r}(\mathbf{x}) d \mathbf{x}
$$

such that

$$
\tilde{D}_{p}^{r}\left(\mathcal{K}_{m}(\mathbf{a}), v\right)=\sup _{B \subset \Omega_{d}: \operatorname{vol}(B)=v}\left\|E_{B}^{r}\right\|_{p}
$$

By formulas (1.6), (1.7) and (2.1) we obtain

$$
E_{B}^{r}(\mathbf{z})=\sum_{\mathbf{k} \neq 0} \hat{\tilde{h}}_{B}^{r}(\mathbf{k}) S(\mathbf{k}, \mathbf{a}) e^{-i 2 \pi(\mathbf{k}, \mathbf{z})}=\sum_{\mathbf{k} \in L(m, \mathbf{a}) \backslash\{0\}} \hat{\tilde{h}}_{B}^{r}(\mathbf{k}) e^{-i 2 \pi(\mathbf{k}, \mathbf{z})} .
$$

It is apparent from (2.4) that it remains to bound $\left\|\delta_{s}\left(E_{B}^{r}\right)\right\|_{p}$.

If $t \neq 0$ is such that $2^{t} \leq L$ then for $\mathbf{s}$ with $\|\mathbf{s}\|_{1}=t$ we have $\rho(\mathbf{s}) \subset$ $\Gamma(L)$. Then our assumption (11.9) implies that $S(\mathbf{k}, \mathbf{a})=0$ for $\mathbf{k} \in \rho(\mathbf{s})$ and, therefore, $\delta_{s}\left(E_{B}^{r}\right)=0$. Let $t_{0} \in \mathbb{N}$ be the smallest number satisfying $2^{t_{0}}>L$, i.e., $t_{0}>\log L$. Then, from (2.4) for $p \in[2, \infty)$, we have

$$
\left\|E_{B}^{r}\right\|_{p} \leq C(p)\left(\sum_{t=t_{0}}^{\infty} \sum_{\|\mathbf{s}\|_{1}=t}\left\|\delta_{s}\left(E_{B}^{r}\right)\right\|_{p}^{2}\right)^{1 / 2}
$$

Moreover, (11.9) implies that for $t \geq t_{0}$ we have

$$
\#(\rho(\mathbf{s}) \cap L(m, \mathbf{a})) \leq C_{2} 2^{t-t_{0}}, \quad\|\mathbf{s}\|_{1}=t .
$$


By Parseval's identity we obtain

$$
\left\|\delta_{s}\left(E_{B}^{r}\right)\right\|_{2}=\sqrt{\sum_{\mathbf{k} \in \rho(\mathbf{s}) \cap L(m, \mathbf{a})}\left|\hat{\tilde{h}}_{B}^{r}(\mathbf{k})\right|^{2}} \leq \sqrt{\#(\rho(\mathbf{s}) \cap L(m, \mathbf{a}))} \cdot H_{B}^{r}(\mathbf{s})
$$

and, by the triangle inequality,

$$
\left\|\delta_{s}\left(E_{B}\right)\right\|_{\infty} \leq \#(\rho(\mathbf{s}) \cap L(m, \mathbf{a})) \cdot H_{B}^{r}(\mathbf{s})
$$

Hence, using the inequality

$$
\|f\|_{p} \leq\|f\|_{2}^{2 / p}\|f\|_{\infty}^{1-2 / p}
$$

for $2 \leq p \leq \infty$, we get

$$
\left\|\delta_{s}\left(E_{B}^{r}\right)\right\|_{p} \leq(\#(\rho(\mathbf{s}) \cap L(m, \mathbf{a})))^{1-1 / p} \cdot H_{B}^{r}(\mathbf{s}) .
$$

Combining this with (2.3), (2.7) and (2.8), we finally obtain for all $v=$ $\operatorname{vol}(B) \geq 2 r^{d} 2^{-t_{0}}$ and $p \in[2, \infty)$ that

$$
\begin{aligned}
\left\|E_{B}^{r}\right\|_{p} & \leq C\left(\sum_{t=t_{0}}^{\infty} 2^{2\left(t-t_{0}\right)(1-1 / p)} \sum_{\|\mathbf{s}\|_{1}=t} H_{B}^{r}(\mathbf{s})^{2}\right)^{1 / 2} \\
& \leq C^{\prime}\left(\sum_{t=t_{0}}^{\infty} 2^{2\left(t-t_{0}\right)(1-1 / p)} 2^{-2 r t}\left(\log \left(2^{t} v\right)\right)^{d-1}\right)^{1 / 2} \\
& =C^{\prime} 2^{-r t_{0}}\left(\sum_{t=0}^{\infty} 2^{2 t(1-1 / p-r)}\left(\log \left(2^{t+t_{0}} v\right)\right)^{d-1}\right)^{1 / 2} \\
& \leq C^{\prime \prime} 2^{-r t_{0}}\left(\log \left(2^{t_{0}} v\right)\right)^{(d-1) / 2}\left(\sum_{t=0}^{\infty} t 2^{2 t(1-1 / p-r)}\right)^{1 / 2}
\end{aligned}
$$

Using $t_{0} \geq \log L$ and that $\left\|E_{B}^{r}\right\|_{p} \leq\left\|E_{B}^{r}\right\|_{2}$ for $p<2$, this implies Theorem 1.4. (Here, we used that clearly $r>1-1 / p$ for $p<\infty$.)

As we pointed out above, in the proof of Theorem 1.5 we use inequality (2.5) instead of (2.4). Moreover we use

$$
\sum_{\|\mathbf{s}\|_{1}=t} H_{B}^{r}(\mathbf{s}) \leq C_{1} 2^{-r t}\left(\log \left(2^{t} v\right)\right)^{d-1}
$$


for all $r \geq 1$ instead of (2.3) . However, note that we need $r>1$ for the last series in the above computation to be finite. This implies

$$
\left\|E_{B}^{r}\right\|_{\infty} \leq C L^{-r}(\log (L v))^{d-1} .
$$

\section{Dispersion of the Korobov point sets}

We remind the definition of dispersion. Let $d \geq 2$ and $[0,1)^{d}$ be the $d$ dimensional unit cube. For $\mathbf{x}, \mathbf{y} \in[0,1)^{d}$ with $\mathbf{x}=\left(x_{1}, \ldots, x_{d}\right)$ and $\mathbf{y}=$ $\left(y_{1}, \ldots, y_{d}\right)$ we write $\mathbf{x}<\mathbf{y}$ if this inequality holds coordinate-wise. For $\mathbf{x}<\mathbf{y}$ we write $[\mathbf{x}, \mathbf{y})$ for the axis-parallel box $\left[x_{1}, y_{1}\right) \times \cdots \times\left[x_{d}, y_{d}\right)$ and define

$$
\mathcal{B}:=\left\{[\mathbf{x}, \mathbf{y}): \mathbf{x}, \mathbf{y} \in[0,1)^{d}, \mathbf{x}<\mathbf{y}\right\} .
$$

For $n \geq 1$ let $T$ be a set of points in $[0,1)^{d}$ of cardinality $|T|=n$. The volume of the largest empty (from points of $T$ ) axis-parallel box, which can be inscribed in $[0,1)^{d}$, is called the dispersion of $T$ :

$$
\operatorname{disp}(T):=\sup _{B \in \mathcal{B}: B \cap T=\emptyset} \operatorname{vol}(B) .
$$

An interesting extremal problem is to find (estimate) the minimal dispersion of point sets of fixed cardinality:

$$
\operatorname{disp}^{*}(n, d):=\inf _{T \subset[0,1)^{d},|T|=n} \operatorname{disp}(T) .
$$

It is known that

$$
\operatorname{disp}^{*}(n, d) \leq C^{*}(d) / n .
$$

Inequality (3.1) with $C^{*}(d)=2^{d-1} \prod_{i=1}^{d-1} p_{i}$, where $p_{i}$ denotes the $i$ th prime number, was proved in [6] (see also [10]). The authors of [6] used the HaltonHammersly set of $n$ points (see [7]). Inequality (3.1) with $C^{*}(d)=2^{7 d+1}$ was proved in [1]. The authors of [1], following G. Larcher, used the $(t, r, d)$ nets (see [8], [7] for results on $(t, r, d)$-nets and Definition 3.1 below for the definition).

Definition 3.1. A $(t, r, d)$-net (in base 2 ) is a set $T$ of $2^{r}$ points in $[0,1)^{d}$ such that each dyadic box $\left[\left(a_{1}-1\right) 2^{-s_{1}}, a_{1} 2^{-s_{1}}\right) \times \cdots \times\left[\left(a_{d}-1\right) 2^{-s_{d}}, a_{d} 2^{-s_{d}}\right)$, $1 \leq a_{j} \leq 2^{s_{j}}, j=1, \ldots, d$, of volume $2^{t-r}$ contains exactly $2^{t}$ points of $T$. 
It was demonstrated in [15] how good upper bounds on fixed volume discrepancy can be used for proving good upper bounds for dispersion. This fact was one of the motivation for studying the fixed volume discrepancy. Theorem 3.1 below was derived from Theorem 1.1 (see [15]). The upper bound in Theorem 3.1 combined with the trivial lower bound shows that the Fibonacci point set provides optimal rate of decay for the dispersion.

Theorem 3.1. There is an absolute constant $C$ such that for all $n$ we have

$$
\operatorname{disp}\left(\mathcal{F}_{n}\right) \leq C / b_{n} .
$$

The reader can find further recent results on dispersion in [11, [12], 20], and 4 .

We now proceed to new results on dispersion of the Korobov point sets.

Theorem 3.2. Suppose that $P_{m}(\cdot, \mathbf{a})$ is exact on $\mathcal{T}(L, d)$ with some $L \in \mathbb{N}$, $L \geq 2$. There exists a constant $C_{1}(d)>0$ such that we have

$$
\operatorname{disp}\left(\mathcal{K}_{m}(\mathbf{a})\right) \leq C_{1}(d) L^{-1} .
$$

Proof. The proof is based on Theorem 1.5. Specify some $r \in \mathbb{N}, r \geq 2$. Then, by Theorem 1.5 we have

$$
\tilde{D}_{\infty}^{r}\left(\mathcal{K}_{m}(\mathbf{a}), v\right) \leq C(d) L^{-r}(\log (L v))^{d-1} .
$$

Let a box $B \in \mathcal{B}$ be an empty box $B \cap \mathcal{K}_{m}(\mathbf{a})=\varnothing$. Denote $v=\operatorname{vol}(B)$. Note that for $B \in \mathcal{B}$ we have $\tilde{h}_{B}^{r}(\mathbf{x})=h_{B}^{r}(\mathbf{x})$. Then, from the Definition 1.2 of the $\tilde{D}_{\infty}^{r}\left(\mathcal{K}_{m}(\mathbf{a}), v\right)$ it follows that

$$
\left|\int_{\Omega_{d}} h_{B}^{r}(\mathbf{x}) d \mathbf{x}-\frac{1}{m} \sum_{\mu=1}^{m} h_{B}^{r}\left(\mathbf{y}^{\mu}\right)\right| \leq \tilde{D}_{\infty}^{r}\left(\mathcal{K}_{m}(\mathbf{a}), v\right) .
$$

Our assumption that $B$ is an empty box implies that

$$
\frac{1}{m} \sum_{\mu=1}^{m} h_{B}^{r}\left(\mathbf{y}^{\mu}\right)=0
$$

and, therefore, by (3.3) we obtain

$$
\left|\int_{\Omega_{d}} h_{B}^{r}(\mathbf{x}) d \mathbf{x}\right| \leq \tilde{D}_{\infty}^{r}\left(\mathcal{K}_{m}(\mathbf{a}), v\right) .
$$


If $v \leq c(d) L^{-1}$, where $c(d)$ is from Theorem 1.5, then Theorem 3.2 with $C_{1}(d) \geq c(d)$ follows. Assume $v \geq c(d) L^{-1}$. Then we apply Theorem 1.5 and obtain from (3.4)

$$
\left|\int_{\Omega_{d}} h_{B}^{r}(\mathbf{x}) d \mathbf{x}\right| \leq C(d) L^{-r}(\log (L v))^{d-1} .
$$

It follows from the definition of functions $h_{B}^{r}(\mathbf{x})$ that

$$
\left|\int_{\Omega_{d}} h_{B}^{r}(\mathbf{x}) d \mathbf{x}\right| \geq c_{1}(d) v^{r} .
$$

Inequalities (3.5) and (3.6) imply that $L v \leq C^{\prime}(d)$.

Setting $C_{1}(d):=\max \left(c(d), C^{\prime}(d)\right)$, we complete the proof of Theorem 3.2 ,

We now make some comments.

Fibonacci point sets. As we already mentioned above we have in the case $d=2, m=b_{n}, \mathbf{a}=\left(1, b_{n-1}\right)$, that $P_{m}(f, \mathbf{a})=\Phi_{n}(f)$. Denote in this case $L(n):=L(m, \mathbf{a})$. In other words

$$
L(n):=\left\{\mathbf{k}=\left(k_{1}, k_{2}\right) \in \mathbb{Z}^{2}: k_{1}+b_{n-1} k_{2} \equiv 0 \quad\left(\bmod b_{n}\right)\right\} .
$$

The following lemma is well known (see, for instance, [17], p.274).

Lemma 3.1. There exists an absolute constant $\gamma>0$ such that for any $n>2$ for the 2-dimensional hyperbolic cross we have

$$
\Gamma\left(\gamma b_{n}, 2\right) \cap(L(n) \backslash\{\mathbf{0}\})=\varnothing .
$$

The combination of Theorem 3.2 with Lemma 3.1 implies Theorem 3.1 .

Special Korobov point sets. Let $L \in \mathbb{N}$ be given. Clearly, we are interested in as small $m$ as possible such that there exists a Korobov cubature formula, which is exact on $\mathcal{T}(L, d)$. In the case of $d=2$ the Fibonacci cubature formula is an ideal in a certain sense choice. There is no known Korobov cubature formulas in case $d \geq 3$, which are as good as the Fibonacci cubature formula in case $d=2$. We now formulate some known results in this direction. Consider a special case $\mathbf{a}=\left(1, a, a^{2}, \ldots, a^{d-1}\right), a \in \mathbb{N}$. In this case we write in the notation of $\mathcal{K}_{m}(\mathbf{a})$ and $P_{m}(\cdot, \mathbf{a})$ the scalar $a$ instead of the vector a, namely, $\mathcal{K}_{m}(a)$ and $P_{m}(\cdot, a)$. The following Lemma 3.2 is a simple well known result (see, for instance [17], p.285). 
Lemma 3.2. Let $m$ and $L$ be a prime and a natural number, respectively, such that

$$
|\Gamma(L, d)|<(m-1) / d .
$$

Then there is a natural number $a \in[1, m)$ such that for all $\mathbf{k} \in \Gamma(L, d)$, $\mathbf{k} \neq \mathbf{0}$

$$
k_{1}+a k_{2}+\cdots+a^{d-1} k_{d} \not \equiv 0 \quad(\bmod m) .
$$

The combination of Theorem 3.2 with Lemma 3.2 implies the following Proposition 3.1.

Proposition 3.1. There exists a positive constant $C_{2}(d)$, which depends only on $d$, with the following property. For any $L \in \mathbb{N}, L \geq 2$, there exist a prime number $m \leq C_{2}(d)|\Gamma(L, d)|$ and a natural number $a \in[1, m)$ such that

$$
\operatorname{disp}\left(\mathcal{K}_{m}(a)\right) \leq C_{1}(d) L^{-1} .
$$

Corollary 3.1. Let $C_{1}(d)$ be the number from Theorem 3.2 and $p$ be a prime number. There exist a natural number $a \in[1, p)$ such that for any segments of natural numbers $I_{1}, \ldots, I_{d} \in[1, p]$ satisfying the condition

$$
\prod_{j=1}^{d}\left|I_{j}\right| \geq C_{1}(d) p^{d-1}(\log p)^{d-1}
$$

there exists a natural number $\mu \in[1, p]$ such that

$$
\left\{\begin{array}{l}
\mu \in I_{1} \quad(\bmod p) \\
\mu a \in I_{2} \quad(\bmod p) \\
\vdots \\
\mu a^{d-1} \in I_{d} \quad(\bmod p) .
\end{array}\right.
$$

Proof. Take

$$
L=\frac{C(d) p}{(\log p)^{d-1}},
$$

where $C(d)$ is small enough to satisfy (3.7). By Lemma 3.2 there exists a natural number $a \in[1, p)$, such that $P_{p}(\cdot, a)$ is exact on $\mathcal{T}(L, d)$.

Denote $I_{j}:=\left[x_{j}, y_{j}\right], j=1, \ldots, d$. Then for $\tilde{I}_{j}:=\left[\frac{x_{j}}{p}, \frac{y_{j}}{p}\right]$ we have

$$
\prod_{j=1}^{d}\left|\tilde{I}_{j}\right| \geq \frac{C_{1}(d)(\log p)^{d-1}}{p} .
$$


By Theorem 3.2 the set $\mathcal{K}_{p}(a)$ intersects the box $\tilde{I}_{1} \times \cdots \times \tilde{I}_{d}$ at least at one point. Then, there exists a natural number $\mu \in[1, p]$, such that one has

$$
\left\{\begin{array}{l}
\left\{\frac{\mu}{p}\right\} \in \tilde{I}_{1}, \\
\left\{\frac{\mu a}{p}\right\} \in \tilde{I}_{2}, \\
\vdots \\
\left\{\frac{\mu a^{d-1}}{p}\right\} \in \tilde{I}_{d} .
\end{array},\right.
$$

which implies (3.9).

Acknowledgment. The work was supported by the Russian Federation Government Grant No. 14.W03.31.0031.

\section{References}

[1] C. Aistleitner, A. Hinrichs, and D. Rudolf, On the size of the largest empty box amidst a point set, Discrete Appl. Math. 230 (2017), 146150.

[2] J. Beck and W. Chen, Irregularities of distribution, Cambridge University Press, Cambridge, 1987.

[3] D. Bilyk, Roth's Orthogonal Function Method in Discrepancy Theory and Some New Connections, in Panorama of Discrepancy Theory, Lecture Notes in Mathematics 2107, Springer-Verlag, London, 2014, $71-158$.

[4] S. Breneis and A. Hinrichs, Fibonacci lattices have minimal dispersion on the two-dimensional torus, preprint, arXiv:1905.03856.

[5] Ding Dũng, V.N. Temlyakov, and T. Ullrich, Hyperbolic Cross Approximation, arXiv:1601.03978v2 [math.NA] 2 Dec 2016.

[6] A. Dumitrescu and M. Jiang, On the largest empty axis-parallel box amidst $n$ points, Algorithmica, 66 (2013), 225-248.

[7] J. Matousek, Geometric Discrepancy, Springer, 1999. 
[8] H. Niederreiter and C. Xing, Low-discrepancy sequences and global function fields with many rational places, Finite Fields Appl., 2 (1996), 241-273.

[9] E. Novak and H. Woźniakowski. Tractability of multivariate problems. Volume II: Standard information for functionals, volume 12 of EMS Tracts in Mathematics. European Mathematical Society (EMS), Zürich, 2010.

[10] G. Rote and F. Tichy, Quasi-Monte Carlo methods and the dispersion of point sequences, Math. Comput. Modelling, 23 (1996), 9-23.

[11] D. Rudolf, An upper bound of the minimal dispersion via delta covers, Contemporary Computational Mathematics - a Celebration of the 80th Birthday of Ian Sloan. Springer-Verlag, 2018.

[12] J. Sosnovec, A note on minimal dispersion of point sets in the unit cube, European J. Combin. 69 (2018), 255259.

[13] V.N. Temlyakov, Approximation of periodic functions, Nova Science Publishers, Inc., New York., 1993.

[14] V.N. Temlyakov, Cubature formulas and related questions, J. Complexity 19 (2003), 352-391.

[15] V.N. Temlyakov, Smooth fixed volume discrepancy, dispersion, and related problems, J. Approx. Theory, 237 (2019), 113-134; arXiv:1709.08158v2 [math.NA] 4 Oct 2017.

[16] V.N. Temlyakov, Fixed volume discrepancy in the periodic case, arXiv:1710.11499v1 [math.NA] 30 Oct 2017.

[17] V.N. Temlyakov, Multivariate approximation, Cambridge University Press, 2018.

[18] V.N. Temlyakov, Connections between numerical integration, discrepancy, dispersion, and universal discretization, arXiv:1812.04489v1 [math.NA] 9 Dec 2018.

[19] V.N. Temlyakov and M. Ullrich, On the fixed volume discrepancy of the Fibonacci sets in the integral norms, arXiv: 1908.04658v1 [math.NA] 13 Aug 2019. 
[20] M. Ullrich, A lower bound for the dispersion on the torus, Math. Comput. Simulation 143 (2018), 186-190.

[21] M. Ullrich, A note on the dispersion of admissible lattices, Discrete Appl. Math. 257 (2019), 385387.

[22] M. Ullrich and J. Vybíral, An upper bound on the minimal dispersion, J. Complexity 45 (2018), 120-126. 